\title{
Ultraviolet Nail Lamps and Squamous Cell Carcinoma: Cause and Effect, or Not?
}

\section{-A Case Report}

\author{
Eva A. Williams ${ }^{1}$, Stuart H. Kuschner ${ }^{2 *}$ \\ ${ }^{1}$ Division of Plastic \& Reconstructive Surgery, Department of Surgery, Keck School of Medicine of the University of Southern \\ California, Los Angeles, USA \\ ${ }^{2}$ Division of Hand Surgery, Department of Orthopedics, Cedars-Sinai Medical Center, Los Angeles, USA \\ Email: eva.williams@med.usc.edu, *stuart.kuschner@cshs.org
}

How to cite this paper: Williams, E.A. and Kuschner, S.H. (2021) Ultraviolet Nail Lamps and Squamous Cell Carcinoma: Cause and Effect, or Not? Open Journal of Orthopedics, 11, 335-339.

https://doi.org/10.4236/ojo.2021.1111032

Received: October 18, 2021

Accepted: November 21, 2021

Published: November 24, 2021

Copyright $\odot 2021$ by author(s) and Scientific Research Publishing Inc. This work is licensed under the Creative Commons Attribution International License (CC BY 4.0).

http://creativecommons.org/licenses/by/4.0/ (c) (i) Open Access

\begin{abstract}
Ultraviolet (UV) lamps are used in nail salons as part of standard gel nail manicures. While UV radiation is a known risk factor for the development of skin cancers, the risk from exposure to UV nail lamps is unclear. We present a case of a woman who developed squamous cell carcinoma in one finger after exposure to UV lamps. A discussion of the correlation between UV nail lamps and squamous cell carcinoma (SCC) is provided. Orthopedic surgeons frequently encounter skin cancers of the hand however the role of UV nail lamps has not yet been elucidated in the orthopedic literature. This manuscript aims to address that gap and facilitate patient counseling during such consultations.
\end{abstract}

\section{Keywords}

Squamous Cell Carcinoma, Hand Surgery, Ultraviolet Light, Cancer Risk

\section{Introduction}

Gel polish is frequently used as part of nail manicures. After it is applied, UV lamps are placed over the nails to cure the polish [1] [2] [3] [4]. Manufacturers state that curing evaporates solvents and establishes strong connections within the polish via acetone-dissolvable polymers [4]. These lamps emit high-intensity UV-A, which is known to increase the risk of skin cancer. UV-A penetrates the skin's dermal layer and causes cellular damage and aging. It is known to be mutagenic, causing oxidative stress and the development of free radicals [1]. For many, there is a concern that gel manicures may cause skin cancer of the hands [5] [6]. 
Gel polish is proprietary and there is minimal regulation of the UV lamps that are used in the curing process. As such, lamp UV-A output varies significantly across manufacturers. Different models have unique curing times and light strength [1].

We report a case of a 59-year-old woman with biopsy-proven SCC. Her hands had been routinely exposed to UV nail lamps for over ten years. We then provide a discussion of a potential relationship between UV nail lamps and the development of SCC.

\section{Case Presentation}

A 59-year-old female was referred for Hand Surgery consultation with two months of right middle finger pain. The pain was associated with color changes in the distal digit. For over a decade her fingers had been exposed to UV light as part of regular gel manicures. The patient, like other gel polish recipients, endorsed use of the light source for curing her nails at the end of nail color application. She did not complain of acute nail changes associated with individual manicures.

On examination, the distal segment of the middle finger was enlarged, and palmar pulp was dusky (Figure 1 and Figure 2). It was exquisitely tender to palpation. The nail plate was loose. X-rays of the middle finger showed osteolysis of the distal tuft (Figure 3).

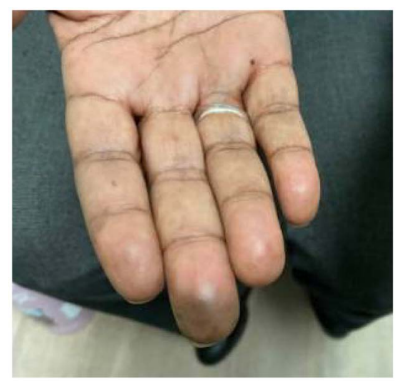

Figure 1. Volar surface of right-hand distal phalanges, notable for duskiness and swelling of the middle finger.

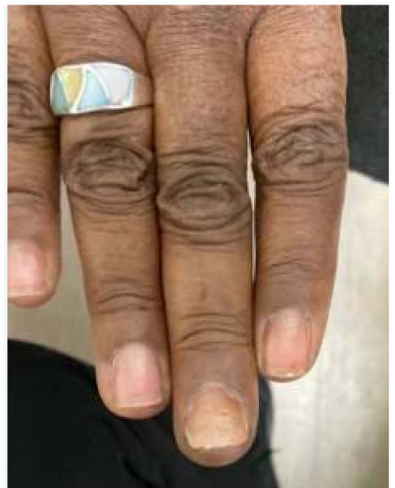

Figure 2. Dorsal surface of right-hand distal phalanges, notable for swelling of the middle finger. 


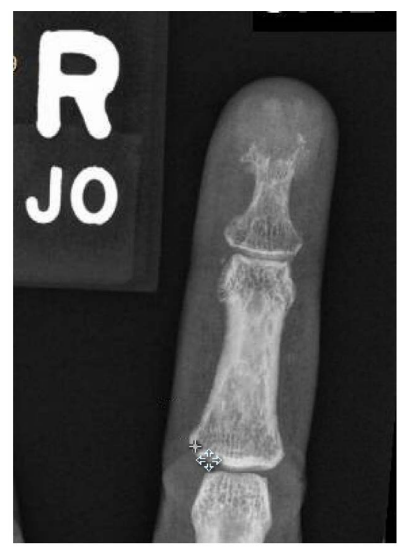

Figure 3. Initial X-ray demonstrating osteolysis of right-hand middle finger distal tuft.

An MRI was obtained which demonstrated osteomyelitis of the distal phalanx. She underwent surgical debridement of the distal segment, with biopsy and culture obtained intra-operatively. Wound cultures were negative for bacterial or fungal growth. Histopathologic examination revealed SCC. The pain did not resolve and two weeks after the initial operation she underwent amputation at the middle finger through the middle phalanx, distal to the insertion at the flexor digitorum superficialis. The surgical specimen showed well-differentiated, invasive SCC with resection margins not involved. Her pain improved and the surgical wound healed without complication. Oncology was consulted and a recommendation was made for follow-up in 6 months. Surveillance imaging was felt to be unnecessary. In retrospect, the patient's unremitting finger pain may have been from ischemia of the distal soft tissue, although the exact cause remains unknown.

As stated previously, there is a concern that a link may exist between the use of UV lights for gel manicures and SCC in the digits. UV radiation is recognized as a carcinogen and well-known risk factor for SCC [7] [8]. Thus, the question arises: is this SCC from gel manicure UV light exposure or is this an example of "true-true, but unrelated?"

\section{Discussion}

In 2009, Macfarlane \& Alonso presented 2 patients with SCC on the dorsum of their hands, each of whom had a history of exposure to UV nail lights [5]. The authors noted that exposure to UV light was a risk factor for the development of skin cancer. Not a controversial statement. Sunlight is the principal factor in skin cancer development and is associated with UV radiation [9]. However, MacFarlane \& Alonso didn't establish a causal relationship between UV nail lamps and skin cancer.

Since the 2009 report by MacFarlane and Alonso other authors have questioned if exposure to UV nail light poses a health risk. Diffey, in 2012, used a mathematical model to compare risk of SCC due to typical sun exposure with risk of SCC from UV nail lamps [10]. Diffey concluded "that tens or hundreds of 
thousands of women would need to use a UVA lamp regularly for one to go on to develop SCC on the dorsum of the hands as a direct consequence." He stated that the risk from UV lamps is one that is likely to be accepted by most individuals.

Curtis et al., in 2013, estimated the cumulative dose of UV nail light over 1 year [10] [11]. They reported that “in less than 10 minutes, a person's hands receive an energy dose equivalent to the day-long recommended limit for outdoor workers." They concluded that long term exposure to UV nail lamps "have the potential" to increase cancer risk.

In contrast, Markova and Weinstock, in 2013, tested 3 commonly used UV nail lamp devices and concluded that they do not appear to significantly increase the lifetime risk of SCC [2]. Shipp et al., in 2014, reported their findings based on random UV light sampling of 17 different light sources from 16 nail salons [12]. Their data "suggest that, even with numerous exposures, the risk for carcinogenesis remains small".

In 2018, Shihab and Lim also concluded that the carcinogenic risk of gel manicures is low however they recommended sunblock or protective gloves before UV lamp exposure [3]. Bollard et al. stated that the "evidence on the carcinogenic risk associated with nail lamps is controversial" and that "further research is warranted" [13].

Ratycz' 2019 case report described a patient with SCC on bilateral dorsal hands [14]. The patient had an 18-year history of UV light exposure at a nail salon. They concluded that "the risk of carcinogenesis from the UV lamps is low" but also stated that UV light "may have been a contributing factor in the development of solar damage to her hands." Talk about hedging one's bets.

Freeman et al., in 2020, presented a 70-year-old female who had been getting regular manicures and pedicures with UVA light exposures [15]. Over a period of 5 years, she developed 7 SCC lesions on the dorsum of fingers and feet (and one SCC on the thigh). The author attributed the distribution of the lesions to the patient's history of UVA light exposure. The authors postulated that since gel nails did not become popular until 2010, the frequency of cases of SCC from gel nail UV light exposure may increase in the coming years.

\section{Conclusion}

At this time, it is impossible to say with absolute certainty that our index patient developed SCC due to UV nail light exposure. However, based on the evidence currently available, orthopedic surgeons can tell their patients that UV lamps are generally safe [1] [3] [6] [11] [12] [13] [14] [15]. The use of sunscreen on the hands and fingerless gloves has been recommended and is an easy and reasonable precaution [11] [12] [13] [14].

\section{Conflicts of Interest}

The authors declare no conflicts of interest regarding the publication of this paper. 


\section{References}

[1] Wang, J.V., Korta, D.Z. and Zachary, C.B. (2018) Gel Manicures and Ultraviolet A Light: A Call for Patient Education. Dermatology Online Journal, 24, 1-2. https://doi.org/10.5070/D3243038625

[2] Markova, A. and Weinstock, M.A. (2013) Risk of Skin Cancer Associated with the Use of UV Nail Lamp. Journal of Investigative Dermatology, 133, 1097-1099. https://doi.org/10.1038/jid.2012.440

[3] Shihab, N. and Lim, H.W. (2018) Potential Cutaneous Carcinogenic Risk of Exposure to UV Nail Lamp: A Review. Photodermatology, Photoimmunology \& Photomedicine, 34, 362-365. https://doi.org/10.1111/phpp.12398

[4] Chen, A.F., Chimento, S.M., Hu, S., et al. (2012) Nail Damage from Gel Polish Manicure. Journal of Cosmetic Dermatology, 11, 27-29. https://doi.org/10.1111/j.1473-2165.2011.00595.x

[5] MacFarlane, D.F. and Alonso, C.A. (2009) Occurrence of Nonmelanoma Skin Cancers on the Hands after UV Nail Light Exposure. Archives of Dermatology, 145, 447-449. https://doi.org/10.1001/archdermatol.2008.622

[6] Schwartz, C.T., Ezaldein, H.H. and Merati, M. (2020) Ultraviolet Light Gel Manicures: Is There a Risk of Skin Cancer on the Hands and Nails of Young Adults? The Journal of Clinical and Aesthetic Dermatology, 13, 45-46.

[7] Powers, J.M. and Murphy, J.E.J. (2019) Sunlight Radiation as a Villain and Hero: 60 Years of Illuminating Research. International Journal of Radiation Biology, 95, 1043-1049. https://doi.org/10.1080/09553002.2019.1627440

[8] Osterlind, A. (1993) Cancer and UV-Radiation. Pharmacology and Toxicology, 72, 67-68. https://doi.org/10.1111/j.1600-0773.1993.tb01672.x

[9] Hernando, B., Dietzen, M., Parra, G., et al. (2021) The Effect of Age on the Acquisition and Selection of Cancer Driver Mutations in Sun-Exposed Normal Skin. Annals of Oncology, 32, 412-421. https://doi.org/10.1016/j.annonc.2020.11.023

[10] Diffey, B.L. (2012) The Risk of Squamous Cell Carcinoma in Women from Exposure to UVA Lamps Used in Cosmetic Nail Treatment. British Journal of Dermatology, 167, 1175-1178. https://doi.org/10.1111/j.1365-2133.2012.11107.x

[11] Curtis, J., Tanner, P., Judd, C., Childs, B., Hull, C. and Leachman, S. (2013) Acrylic Nail Curing UV Lamps: High-Intensity Exposure Warrants Further Research of Skin Cancer Risk. Journal of the American Academy of Dermatology, 69, 1069-1070. https://doi.org/10.1016/j.jaad.2013.08.032

[12] Shipp, L.R., Warner, C.A., Rueggeberg, F.A. and Davis, L.S. (2014) Further Investigation into the Risk of Skin Cancer Associated with the Use of UV Nail Lamps. JAMA Dermatology, 150, 775-776. https://doi.org/10.1001/jamadermatol.2013.8740

[13] Bollard, S.M., Beecher, S.M., Moriarty, N., et al. (2018) Skin Cancer Risk and Use of UV Nail Lamps. Australasian Journal of Dermatology, 59, 348-349. https://doi.org/10.1111/ajd.12806

[14] Ratycz, M.C., Lender, J.A. and Gottwald, L.D. (2019) Multiple Dorsal Hand Actinic Keratoses and Squamous Cell Carcinomas: A Unique Presentation Following Extensive UV Nail Lamp Use. Case Reports in Dermatology, 11, 286-291. https://doi.org/10.1159/000503273

[15] Freeman, C., Hull, C., Sontheimer, R. and Curtis, J. (2020) Squamous Cell Carcinoma of the Dorsal Hands and Feet after Repeated Exposure to Ultraviolet Nail lamps. Dermatology Online Journal, 26, 1-3. https://doi.org/10.5070/D3263047974 